\title{
Self-Reported Opioid Use and Driving Outcomes among Older Adults: The AAA LongROAD Study
}

\author{
Marian E. Betz, MD, MPH, Hailey Hyde, MPH, Carolyn DiGuiseppi, MD, PhD, MPH, \\ Timothy F. Platts-Mills, MD, MSc, Jason Hoppe, DO, David Strogatz, PhD, \\ Howard F. Andrews, PhD, Thelma J. Mielenz, PT, PhD, MS, Linda L. Hill, MD, MPH, \\ Vanya Jones, PhD, MPH, Lisa J. Molnar, PhD, David W. Eby, PhD, and \\ Guobua Li, DrPH, MD
}

Background: Opioid medications are important therapeutic options to mitigate the harmful effects of pain but can also impair driving ability. We sought to explore opioid use, pain levels, and driving experiences among older drivers.

Methods: Cognitively intact drivers ages 65 to 79 years were recruited for the multisite AAA Longitudinal Research on Aging Drivers (LongROAD) study $(\mathrm{n}=2990)$. This cross-sectional analysis used data from the baseline questionnaire and "brown-bag" medication review.

Results: Among LongROAD participants (47\% male, 88\% white, $41 \%$ aged 65 to 69 years), 169 (5.7\%) reported currently taking an opioid, with a median daily dose of 20 morphine milligram equivalents. Participants did not differ significantly in opioid use by age, gender, race, or ethnicity $(P>.05)$. After adjustment for age, gender, race and ethnicity, participants who were taking opioids (vs not) were significantly more likely to report self-regulated driving reduction and reduced driving ability. However, these effects became nonsignificant when hospitalization, impaired physical function and other factors associated with opioid use were controlled.

Conclusions: In this study from a large, geographically diverse sample of older adults, there was an association between opioid use and several self-reported measures of driving behavior and ability. However, future work should clarify the effects on driving of opioid use from the effects of the painful medical conditions for which the opioids are being taken. Clinicians should continue to discuss the risks and benefits of opioid medications with patients, including risks related to driving safety. ( $\mathrm{J}$ Am Board Fam Med 2020;33:521-528.)

Keywords: Automobile Driving, Cross-Sectional Studies, Opioid-Related Disorders, Opioids, Pain, Risk Assessment, Surveys and Questionnaires

\section{Introduction}

Opioid safety and efficacy for the treatment of chronic noncancer pain remains an important and contentious topic in the United States. This may be

This article was externally peer reviewed.

Submitted 20 November 2019; revised 10 January 2020; accepted 13 January 2020.

From the Department of Emergency Medicine, University of Colorado School of Medicine, Aurora (MEB, $\mathrm{JH}$ ); Department of Epidemiology, Colorado School of Public Health, University of Colorado Anschutz Medical Campus, Aurora (HH, CD); Department of Emergency Medicine, University of North Carolina School of Medicine, Chapel Hill (TFPM); Bassett Research Institute, Cooperstown, NY (DS); Department of Psychiatry, College of Physicians and Surgeons, Columbia University, New York, NY (HFA); Department of Biostatistics, Mailman School of Public Health, Columbia University, New York, particularly true for older adults, who report pain as the most common symptom underlying disability ${ }^{1,2}$ but also have unique risk-benefit considerations (eg, polypharmacy, drug metabolism, comorbidities) for

NY (HFA); Department of Epidemiology, Mailman School of Public Health, Columbia University, New York, NY (TJM, GI); Department of Family Medicine and Public Health, University of California-San Diego, La Jolla (LLH); Department of Health, Behavior and Society, Bloomberg School of Public Health, Johns Hopkins University, Baltimore, MD (VJ); University of Michigan Transportation Research Institute and the Center for Advancing Transportation Leadership and Safety (ATLAS Center), Ann Arbor (LJM, DWE); Department of Anesthesiology, Columbia University-Vagelos College of Physicians and Surgeons, New York, NY (GI).

Funding: This work was supported by the AAA Foundation for Traffic Safety (Washington, DC) and by a 
opioid use compared with younger people. ${ }^{3}$ Moreover, there has been a marked increase in both the prescribed dose and number of opioid prescriptions from office-based visits for older adults over the past few decades, ${ }^{1,3}$ reflecting either an increased need for and acceptance of pain management or other shifting trends in opioid prescribing practices.

Older adults face increased risk of experiencing chronic pain due to the aging process and paininducing diseases. ${ }^{4}$ Approximately $25 \%$ to $50 \%$ of adults $\geq 65$ years of age experience pain, typically in multiple sites and for various reasons, including musculoskeletal conditions and cancer. ${ }^{5,6}$ Pain may not only interfere with daily activities, ${ }^{7}$ but may also decrease quality of life for older adults. ${ }^{8,9}$ Chronic pain can also affect mobility-a major predictor of wellbeing ${ }^{10}$ - through effects on cognition (eg, attentional capacity, processing speed, psychomotor speed $)^{11}$ or physical function. One study found that older adults experiencing chronic pain not only had difficulties with driving performance, but also with the physical process of getting into and positioning themselves in a vehicle. ${ }^{12}$ Some with chronic pain may choose to reduce or adapt their driving, ${ }^{13,14}$ which may have negative impacts such as loss of independence, depression, or decreased life satisfaction. ${ }^{15,16}$ Few studies have explored how pain affects driving "self-regulation," the process of adapting one's driving by driving less or avoiding situations considered challenging due to declines in functioning.

Treating acute and chronic pain with medications or other modalities is important to mitigate these negative effects on wellbeing and mobility. Prescription opioid medications are often used to treat pain unresponsive to over-the-counter options. Over the past decade, there has been growing concern over the increases in morbidity and mortality from prescription opioid use in the

Paul Beeson Career Development Award (The National Institute on Aging; AFAR; The John A. Hartford Foundation; and The Atlantic Philanthropies; Grant Number Betz K23AG043123), and the National Center for Injury Prevention and Control of the Centers for Disease Control and Prevention (Grant 1 R49 CE002096). The contents of the manuscript are solely the responsibility of the authors and do not necessarily reflect the official views of the funding agencies.

Conflicts of interest: None.

Corresponding author: Marian E. Betz, MD, MPH, University of Colorado School of Medicine, 12401 E. 17th Ave B-215; Aurora, CO 80045 (E-mail: marian.betz@ ucdenver.edu).
United States. In 2017, the US federal government declared the opioid epidemic a national public health emergency. ${ }^{17}$ Older adults comprise an important population group for research on opioid use because they often use pain medications to manage their chronic pain and maintain wellbeing. ${ }^{5}$

Unfortunately, pain medications themselves may be detrimental to driving safety. ${ }^{18,19}$ In particular, increased crash risk has been linked to use of opioids, ${ }^{20,21}$ along with benzodiazepines, opioid antagonists, and other medications related to pain management. ${ }^{22,23}$ Use of multiple medications that act on the central nervous system (ie, CNS polypharmacy) is of particular concern in older adults and is included in the Beers Criteria for Potentially Inappropriate Medications. ${ }^{24}$ Yet, because decreased mobility due to chronic pain may lead to decreased wellbeing, simply restricting access to pain medications is not necessarily the answer.

The AAA Longitudinal Research on Aging Drivers (LongROAD) study presents the opportunity to examine opioid use in a large cohort of older drivers, and importantly, to consider its relationship to self-reported measures of pain, self-regulation of driving, motor vehicle crashes, and self-rated driving ability and comfort. In this analysis, we sought to determine, in a sample of older drivers 1) the prevalence of opioid use; 2) the prevalence and severity of daily pain; and 3) the associations between opioid use, pain levels, and self-reported driving behaviors. Understanding these relationships may inform programs and prescribing practices to better address health, mobility, and quality of life.

\section{Methods}

\section{Design and Participants}

This cross-sectional analysis used baseline data from the AAA LongROAD study, a multi-site, prospective cohort study designed to evaluate the role of medical, behavioral, and other factors in driving safety in older adults. ${ }^{25}$ Participants were recruited from health care clinics affiliated with 5 LongROAD sites. Eligible patients were aged 65 to 79 years at enrollment, spoke English, had a valid driver's license, drove at least 1 day per week, drove 1 motor vehicle (model 1996 or newer with an accessible on board diagnostics [OBDII] port) at least $80 \%$ of the time, resided in the site catchment area at least 10 months a year with no plans to move outside the catchment area within the next 5 years, and passed a 6-item screener 
for cognitive function. ${ }^{26}$ Informed consent was obtained during the baseline visit by research staff, and the study was approved by each site's institutional review board. The study design and population have been described in detail elsewhere. ${ }^{25}$

\section{Measures and Analysis}

Data included self-reported demographic and health characteristics, functional abilities, health care utilization, medication use, and subjective measures of driving collected by research staff at enrollment. Self-reported demographic characteristics included age group, gender, race, ethnicity, highest level of educational attainment, and total household income in the past year. Self-reported health domains were measured by the PatientReported Outcomes Measurement Information System (PROMIS), including Physical Function-4a and Pain Intensity. ${ }^{27}$ PROMIS Physical Function T-scores were trichotomized as "none to slight limited function" ( $>55)$, "mild limited function" (40 to $55)$, or "moderate to severe limited function" $(<40)$. PROMIS Numeric Rating Scale v1.0-Pain Intensity $1 \mathrm{~A}$ was measured by self-reported average pain in the past 7 days, ranging from "no pain" (0) to "worst imaginable pain" (10); for analyses, responses were categorized into 4 groups: "none" (0), "mild" (1 to 3), "moderate" (4 to 6), "severe" (7 to 10). Additional self-reported measures of health behaviors included alcohol consumption in the past 3 months (Yes/No) and health care utilization in the past 12 months (Yes/No).

Information on current medications (prescribed and over-the-counter) and supplements was collected using a "brown-bag review" method at baseline. Forty-one LongROAD participants (1.4\%) without baseline medication review were excluded. For analysis, medications were categorized according to their American Hospital Formulary System classification. Individuals were categorized as currently taking an opioid if they reported taking 1 or more opiate agonists (codeine, fentanyl, oxycodone, tramadol, hydrocodone, hydromorphone, methadone, morphine). Consistent with the US Centers for Disease Control and Prevention (CDC) guidelines, morphine milligram equivalents (MME) per day were calculated by multiplying the total daily dose of each opioid by the requisite conversion factor. ${ }^{28}$ For participants taking more than 1 opioid, MMEs were summed to obtain the total daily MME. Doses listed as PRN were calculated using the maximum prescribed dose and frequency per day.

The 5 self-reported driving outcomes included driving ability; driving comfort; driving reduction due to self-regulation; driving lapses, errors, and violations; and crashes or police actions in the past year. Self-rated driving ability averaged participants' responses to 5 items (ability to see during the day, to see at night, to remember things, to concentrate on more than 1 thing at time, and their strength, flexibility, and general mobility) rated from "poor" (1) to "excellent" (7). Self-rated driving comfort averaged participants' responses to multiple items assessing comfort in potentially difficult driving conditions (eg, driving at night, in bad weather, alone, on the freeway), ranging from "not at all comfortable" (1) to "completely comfortable" (7). Participants were asked whether they had reduced their driving in the last year $(\mathrm{Yes} / \mathrm{No})$ and, if so, if the reduction was due to self-regulation (Yes/No), that is, because of difficulty seeing during the day or night; difficulty remembering things; difficulty concentrating on more than 1 thing at a time; not feeling safe driving as much; not feeling comfortable driving as much; experiencing reductions in strength, flexibility, or general mobility; or other reasons related to declines in functioning. These 2 questions were used to ascertain self-regulated driving reduction (Yes/No). To assess driving lapses, errors, and violations, participants completed the Driving Behavior Questionnaire, ${ }^{24-28}$ with 6 questions pertaining to lapses (eg, forgetting where you left your car in a parking lot), 9 to errors (eg, failing to check your rear-view mirror before pulling out, changing lanes), and 11 to violations (eg, crossing an intersection knowing the light has already turned red). Each question was scaled from "never" (0) to "nearly all the time" (5). The mean of each was used in analysis, with higher scores indicating more lapses, errors, or violations (ie, worse driving). "Crashes or police actions" (Yes/ No) were based on participant self-report of having had a crash or police action (ie, being pulled over by police or receiving a traffic ticket) while driving in the past year.

Characteristics of the study sample were described with means and standard deviations for continuous variables and frequency distributions for categorical variables. Chi-Square tests (or Fisher's exact tests) for categorical variables, or $t$ test for continuous variables, were used to assess 
each covariate's association with opioid use and each driving outcome. The unadjusted associations between opioid use and each self-reported measure of driving were examined using linear or logistic regression, as appropriate.

Partially adjusted models for each self-reported driving outcome and opioid use were utilized to account for potential differences in age, gender, race, and ethnicity. Covariates which were associated with opioid use and self-reported driving outcomes $(P<.2)$ were considered in multivariable models using forward selection. The model with the lowest Akaike information criterion was selected as the final model for each self-reported driving outcome. All models used complete case analysis. Results are reported as $\beta$ estimates or odds ratios, with $95 \%$ CIs, using an $\alpha$ level of 0.05 for testing statistical significance. Model assumptions and fit were assessed using residuals, adjusted R-square, probability plots, and Akaike information criterion as applicable. All analyses were conducted using SAS University Edition software (version 9.04.01, SAS Institute, Inc., Cary, NC).

\section{Results}

Of the 2949 LongROAD participants with medication data, $169(5.7 \%)$ reported currently taking an opioid (Table 1); these participants had a higher self-reported level of pain in the past 7 days (5.1 vs 3.8 out of $10, P<.001)$ and a median reported dose of 20 MME per day. Most participants were 65 to 69 years of age $(41.6 \%)$, female $(53.1 \%)$, white (88.9\%), and non-Hispanic (97.2\%), with no significant differences in the prevalence of opioid use based on age, gender, race, or ethnicity. However, the percentage taking an opioid was more than twice as high among those without a Bachelor's degree $(8.9 \%)$ than among those with a Bachelor's or higher degree $(4.2 \%$ and $3.9 \%$ respectively; $P<.001)$. Similarly, opioid use was much higher among those with household income less than $\$ 50,000$ per year $(10.2 \%)$ than among those with household income greater than $\$ 100 \mathrm{k}$ per year (3.1\%; $P<.001)$. As might be expected, opioid use was significantly higher among those reporting emergency department visits $(P<.001$ or hospitalizations $(P<.001)$ in the past year, and among those who had spoken to a physician during the past year about driving safety $(P<.001)$. Opioid use was more than 10 times greater among those reporting moderate to severe problems with physical function $(21.7 \%)$ than among those reporting no or mild limitations $(1.7 \% ; P<.0001)$. However, opioid use was significantly lower among those who reported any versus no alcohol use in the past 3 months ( $4.9 \%$ versus $8.0 \%, P=.002$ ).

In unadjusted analyses, participants who reported current opioid use were more likely than those not using opioids to report self regulating their driving (11.2\% versus $5.4 \%, P=.002$; Table 2 and Table 3). Opioid users also rated their driving ability lower than nonusers, 5.7 versus 5.9 out of 7 ["excellent"], $P<.001)$. There was a $5.4 \%$ absolute increase in reported crashes or police action in the last year among opioid users versus nonusers (28.4\% vs $23.0 \%)$. No statistically significant differences were seen in subset sensitivity analyses by low and high MME dose groups or by PRN and standing dosage groups (data not shown).

Adjusting for age, gender, and race/ethnicity did not significantly alter these main findings (Table 2 and Table 3). However, the findings did change after additional adjustment for income, education, emergency department visits, hospitalizations, alcohol use, and physical function. Specifically, there was no longer a statistically significant difference in self-regulated driving reduction or self-rated driving ability by opioid use. However, in this fully adjusted model, individuals who reported current opioid use had fewer self-reported driving lapses $(P=.027)$ and higher self-rated driving comfort $(P=.009)$ than individuals who reported no opioid use, although the mean group differences on both 2 scales were quite small.

\section{Discussion}

In the context of debates about optimizing older adult function and mobility while avoiding the negative effects of opioid use, this study sought to investigate the relationships between opioid use, pain, and driving in a large sample of older drivers. We found that only $5.7 \%$ of older drivers reported currently using an opioid, and opioid use varied by education and income (though not by age, gender, or race/ethnicity). Opioid users had higher rates of self-regulated driving reduction and lower selfrated driving ability, along with a higher (though not significantly so) rate of crashes or police actions. These differences disappeared after adjustment for demographic and health characteristics, highlighting 


\begin{tabular}{|c|c|c|c|c|c|c|}
\hline & \multicolumn{2}{|c|}{$\begin{array}{l}\text { Any Opioid Use } \\
(\mathrm{n}=169)\end{array}$} & \multicolumn{2}{|c|}{$\begin{array}{c}\text { No Opioid Use } \\
(\mathrm{n}=2780)\end{array}$} & \multirow{2}{*}{$\begin{array}{l}\text { Prevalence } \\
\text { Ratio }\end{array}$} & \multirow[b]{2}{*}{$95 \% \mathrm{CI}$} \\
\hline & $\mathrm{n}$ & $\%$ & $\mathrm{n}$ & $\%$ & & \\
\hline \multicolumn{7}{|l|}{ Age Group (years) } \\
\hline 65 to 69 & 69 & 5.6 & 1153 & 94.4 & - & - \\
\hline 70 to 74 & 60 & 5.8 & 967 & 94.2 & 1.04 & $0.73,1.48$ \\
\hline 75 to 79 & 40 & 5.7 & 660 & 94.3 & 1.01 & $0.68,1.51$ \\
\hline \multicolumn{7}{|l|}{ Gender } \\
\hline Male & 73 & 5.3 & 1309 & 94.7 & 0.86 & $0.63,1.17$ \\
\hline Female & 96 & 6.1 & 1471 & 93.9 & - & - \\
\hline \multicolumn{7}{|l|}{ Race } \\
\hline White/Caucasian & 143 & 5.5 & 2440 & 94.5 & - & - \\
\hline Nonwhite & 22 & 6.9 & 297 & 93.1 & 1.26 & $0.79,2.01$ \\
\hline \multicolumn{7}{|l|}{ Ethnicity } \\
\hline Hispanic/Latino & 5 & 6.3 & 75 & 93.7 & 1.12 & $0.45,2.81$ \\
\hline Non-Hispanic/Latino & 155 & 5.6 & 2600 & 94.4 & - & - \\
\hline \multicolumn{7}{|l|}{ Highest level of education } \\
\hline Less than bachelor's degree & 93 & 8.9 & 949 & 91.1 & 2.42 & $1.69,3.47$ \\
\hline Bachelor's degree & 29 & 4.2 & 662 & 95.8 & 1.08 & $0.68,1.74$ \\
\hline Master's or higher degree & 47 & 3.9 & 1161 & 96.1 & - & - \\
\hline \multicolumn{7}{|l|}{ Total household income past year } \\
\hline Less than $\$ 50,000$ & 78 & 10.2 & 686 & 89.8 & - & - \\
\hline$\$ 50000$ to $<\$ 80,000$ & 42 & 5.9 & 668 & 94.1 & 0.55 & $0.38,0.82$ \\
\hline$\$ 80,000$ to $<\$ 100,000$ & 17 & 4.0 & 410 & 96.0 & 0.37 & $0.21,0.63$ \\
\hline$\$ 100,000$ or more & 29 & 3.1 & 913 & 96.9 & 0.28 & $0.18,0.43$ \\
\hline \multicolumn{7}{|l|}{ ED visit in the past year } \\
\hline None & 98 & 4.3 & 2161 & 95.7 & - & - \\
\hline At least one & 71 & 10.5 & 607 & 89.5 & 2.58 & $1.88,3.55$ \\
\hline \multicolumn{7}{|l|}{ Hospital visit in the past year } \\
\hline None & 115 & 4.6 & 2371 & 95.4 & - & - \\
\hline At least one & 53 & 11.8 & 398 & 88.2 & 2.74 & $1.95,3.87$ \\
\hline \multicolumn{7}{|l|}{ Ever spoken to doctor about driving safety } \\
\hline No & 145 & 5.1 & 2675 & 94.9 & - & - \\
\hline Yes & 24 & 14.5 & 141 & 85.5 & 3.14 & $1.98,4.99$ \\
\hline \multicolumn{7}{|l|}{ Alcohol use past 3 months } \\
\hline None & 64 & 8.0 & 738 & 92.0 & 1.68 & $1.22,2.33$ \\
\hline Any & 105 & 4.9 & 2041 & 95.1 & - & - \\
\hline $4+$ drinks on one occasion past 3 months & 12 & 4.1 & 295 & 95.9 & 0.65 & $0.36,1.18$ \\
\hline \multicolumn{7}{|l|}{ Limited physical function } \\
\hline None to slight (T-score > 55) & 27 & 1.7 & 1548 & 98.3 & - & - \\
\hline Mild (T-score 40 to 55) & 88 & 7.9 & 1020 & 92.1 & 4.95 & $3.19,7.67$ \\
\hline Moderate to severe $(T$-score $<40$ ) & 52 & 21.7 & 188 & 78.3 & 15.86 & $9.73,25.86$ \\
\hline \multicolumn{7}{|l|}{ Average pain past 7 days } \\
\hline 0 & 29 & 1.9 & 1510 & 98.1 & - & - \\
\hline 1 to 3 & 37 & 5.6 & 623 & 94.4 & 3.09 & $1.89,5.07$ \\
\hline 4 to 6 & 70 & 12.2 & 506 & 87.8 & 7.20 & $4.62,11.23$ \\
\hline 7 to 10 & 33 & 19.3 & 138 & 80.7 & 12.45 & $7.34,21.12$ \\
\hline Morphine milligram equivalents per day, median (IQR) & 20.0 & 20.0 & - & - & - & - \\
\hline
\end{tabular}

AAA, American automobile association; LongROAD, Longitudinal research on aging drivers; ED, emergency department; CI, confidence interval. 
Table 2. Associations between Opioid Use and Self-Reported Driving Outcomes

\begin{tabular}{|c|c|c|c|c|c|c|c|c|c|c|}
\hline & \multicolumn{2}{|c|}{$\begin{array}{c}\text { Any Opioid Use } \\
(\mathrm{n}=169) \\
\end{array}$} & \multicolumn{2}{|c|}{$\begin{array}{l}\text { No Opioid Use } \\
(\mathrm{n}=2821) \\
\end{array}$} & \multicolumn{2}{|c|}{$\begin{array}{c}\text { Unadjusted } \\
\text { OR }\end{array}$} & \multicolumn{2}{|c|}{$\begin{array}{c}\text { Partially } \\
\text { Adjusted OR } \\
\end{array}$} & \multicolumn{2}{|c|}{$\begin{array}{c}\text { Fully } \\
\text { Adjusted OR* }\end{array}$} \\
\hline & $\mathrm{n}$ & $\%$ & $\mathrm{n}$ & $\%$ & OR & $95 \%$ CI & AOR & $95 \%$ CI & AOR & $95 \% \mathrm{CI}$ \\
\hline \multicolumn{11}{|c|}{$\begin{array}{l}\text { Self-regulated driving } \\
\text { reduction }\end{array}$} \\
\hline Yes & 19 & 11.2 & 151 & 5.4 & 2.21 & $1.33,3.66$ & 2.38 & $1.42,3.99$ & $0.99^{\text {adg }}$ & $0.55,1.80$ \\
\hline No & 149 & 88.2 & 2615 & 94.1 & - & - & - & - & - & - \\
\hline \multicolumn{11}{|c|}{$\begin{array}{l}\text { Any crash or police } \\
\text { action in past year }\end{array}$} \\
\hline Yes & 48 & 28.4 & 640 & 23.0 & 1.31 & $0.94,1.87$ & 1.34 & $0.93,1.91$ & $1.25^{\mathrm{bcg}}$ & $0.86,1.83$ \\
\hline No & 120 & 71.0 & 2119 & 76.2 & - & - & - & - & - & - \\
\hline
\end{tabular}

AOR, adjusted odds ratio; OR, odds ratio; CI, confidence interval.

${ }^{*}$ In addition to age, gender, race and ethnicity, models may be adjusted for ${ }^{\text {a }}$ total household income, bhighest level of education, ${ }^{c}$ emergency department visit in the past year; ${ }^{d}$ hospital visit in the past year, ${ }^{e}$ alcohol use in the past 3 months, ${ }^{\mathrm{f}}$ had 4 or more drinks on 1 occasion in the past 3 months, ${ }^{\mathrm{g}}$ physical function.

the complexity of the relationship between pain, opioid use, and driving among older adults. This cross-sectional analysis generates additional questions for future study as well as key considerations for clinicians caring for older adults.

In the LongROAD study cohort, only a small proportion of older drivers reported current opioid use $(5.7 \%)$, despite nearly half of all participants reporting at least mild pain in the past 7 days. According to the CDC, $26.8 \%$ of adults over 65 years of age in the United States filled at least 1 prescription for an opioid in 2017, a higher proportion than any other age group. ${ }^{29}$ The lower proportion of self-reported opioid use in this LongROAD cohort may reflect the different time frame (current vs annual) or a healthier cohort compared with average individuals over 65 years of age in the United States. Despite individuals with higher selfreported pain intensity being more likely to report taking an opioid than individuals who reported no pain in the past 7 days, self-reported levels of pain were comparable to nationally reported experiences of pain in this age group. ${ }^{2}$ There has been growing evidence that opioid efficacy for treating chronic pain is not always sustained over the long-term and that the risks garnered by long-term use (eg, drug dependency or injury) in older adults are serious. ${ }^{30}$ However, unmanaged pain can lead to sleep disturbance, impaired quality of life, disability, decreased mobility, and depression, ${ }^{31}$ so these decisions necessitate patient-centered shared decision making about the relative risks and benefits.

Table 3. Associations between Opioid Use and Self-Reported Driving Outcomes

\begin{tabular}{|c|c|c|c|c|c|c|c|c|c|c|}
\hline & \multirow[b]{2}{*}{ Mean } & \multirow[b]{2}{*}{ SD } & \multirow[b]{2}{*}{ Mean } & \multirow[b]{2}{*}{ SD } & \multicolumn{2}{|c|}{$\begin{array}{c}\text { Unadjusted Beta } \\
\text { Estimate }\end{array}$} & \multicolumn{2}{|c|}{$\begin{array}{c}\text { Partially Adjusted } \\
\text { Beta Estimate* }\end{array}$} & \multicolumn{2}{|c|}{$\begin{array}{l}\text { Fully Adjusted } \\
\text { Beta Estimate }\end{array}$} \\
\hline & & & & & $\beta$ & $95 \% \mathrm{CI}$ & $\beta$ & $95 \% \mathrm{CI}$ & B & $95 \% \mathrm{CI}$ \\
\hline $\begin{array}{l}\text { Self-rated driving } \\
\text { ability }\end{array}$ & 5.7 & 0.7 & 5.9 & 0.7 & -0.18 & $-0.29,-0.08$ & -0.19 & $-0.30,-0.08$ & $0.04^{\mathrm{ag}}$ & $-0.08,0.15$ \\
\hline $\begin{array}{l}\text { Self-rated driving } \\
\text { comfort }\end{array}$ & 5.8 & 0.9 & 5.8 & 1.0 & -0.02 & $-0.17,0.13$ & $<0.00$ & $-0.15,0.15$ & $0.21^{\mathrm{adg}}$ & $0.05,0.36$ \\
\hline Lapses & 1.8 & 0.4 & 1.8 & 0.4 & -0.03 & $-0.09,0.04$ & -0.04 & $-0.11,0.03$ & $-0.08^{\text {befg }}$ & $-0.15,-0.01$ \\
\hline Errors & 1.4 & 0.3 & 1.4 & 0.3 & $<0.00$ & $-0.04,0.06$ & $<0.00$ & $-0.05,0.06$ & $-0.03^{\text {befg }}$ & $-0.09,0.02$ \\
\hline Violations & 1.6 & 0.4 & 1.6 & 0.4 & -0.02 & $-0.08,0.04$ & -0.03 & $-0.10,0.03$ & $-0.02^{\mathrm{abef}}$ & $-0.08,0.04$ \\
\hline
\end{tabular}

*Adjusted for age, gender, race, and ethnicity.

${ }^{\dagger}$ In addition to age, gender, race and ethnicity, models may be adjusted for ${ }^{\text {a }}$ total household income, ${ }^{b}$ highest level of education, cemergency department visit in the past year; ${ }^{\mathrm{d}}$ hospital visit in the past year, ${ }^{\mathrm{e}}$ alcohol use in the past 3 months, ${ }^{\mathrm{f}}$ had 4 or more drinks on 1 occasion in the past 3 months, ${ }^{g}$ physical function.

$\mathrm{SD}$, standard deviation; CI, confidence interval. 
While use of prescription opioids by drivers has been found to be associated with increased risk of crash and crash culpability, ${ }^{20}$ particularly due to sedating effects, ${ }^{23}$ self-reported measures of driving differed very little by self-reported opioid use in this study population. Older drivers who reported currently taking an opioid were more likely to selfregulate and reduce their driving and to report lower self-rated driving ability, regardless of age, gender, or race/ethnicity. These differences disappeared after further adjustment for other demographic and health variables, including health care utilization and physical function, suggesting that the indication for pain management may affect these driving outcomes more than the opioid use itself. The full model was selected after stepwise examination of associations with opioid use and each outcome, but questions remain concerning the relationships of these potential confounders with the outcome. This complex relationship likely reflects the real-life influences of social determinants of health, with socioeconomic factors affecting mobility and health both directly and indirectly. Older adults with lower incomes may be more likely to use opioids in part because of lesser ability to access alternative pain control modalities such as massage, physical therapy, and acupuncture. It is unclear whether driving ability or safety would differ by opioid use on objective measures of driving, but there is little reason to suspect that self-reporting of these driving outcomes was significantly biased by opioid use.

It is important to note that the median daily dose seen in this study was $20 \mathrm{MME}$, much lower than the average daily dose in the total US population (45.3 MME), ${ }^{29}$ which may also explain the lack of an association between opioid use and reported crashes or police actions. Since driving impairments may be greatest soon after starting opioid treatment or at higher doses than seen in this study, prescribing practices (eg, starting low and increasing slowly, avoiding other sedating medications or substances) and prescriber counseling (eg, to avoid driving during the first week) may mitigate the most significant risks to driving from opioid use. ${ }^{23}$ Given the importance of driving to mobility and independence for many older adults, even a $5 \%$ reduction in driving could be clinically meaningful — as could the potential $5 \%$ increase in crashes or police actions. Thus, while opioids remain an important therapeutic option for older adults with severe pain, our findings should not be interpreted to mean they are without risk.

Limitations of this study include reliance on selfreport of both exposures and outcomes, as well as a lack of detail about opioid use when prescribed $\mathrm{PRN}$ and timing of use in relation to driving). The LongROAD cohort, recruited in 5 states, comprises generally healthy, educated older drivers and may not be representative of the general population. Because of the low prevalence of opioids use, the study did not have sufficient power to examine specific types of opioids or polypharmacy. Future work should address these issues.

\section{Conclusion}

Driving remains an essential part of independence, mobility, wellbeing, and maintaining social connectedness. In this large, geographically diverse sample of older drivers, those currently taking an opioid were more likely to report self-regulated reduction of driving, regardless of age, gender, or race/ethnicity. These differences disappeared after further adjustment (including for socioeconomic characteristics, health and health care utilization), but they suggest that older adults using opioids may themselves try to mitigate the negative effects of these medications. Clinicians caring for older adults who use-or are considering initiating-opioids should continue to discuss the risks and benefits of these medications, along with recommendations to avoid driving immediately after taking opioids.

We appreciate the contributions of Barbara Lang to overall study management and of Alexandra Smith and Deepika Kandasamy to analysis and manuscript development. We also gratefully acknowledge the efforts of the LongROAD study coordinators, research assistants, and participants.

To see this article online, please go to: http://jabfm.org/content/ 33/4/521.full.

\section{References}

1. West NA, Severtson SG, Green JL, Dart RC. Trends in abuse and misuse of prescription opioids among older adults. Drug Alcohol Depend 2015;149:117-21.

2. Patel KV, Guralnik JM, Dansie EJ, Turk DC. Prevalence and impact of pain among older adults in the United States: findings from the 2011 National Health and Aging Trends Study. Pain 2013;154:2649-57. 
3. Steinman MA, Komaiko KDR, Fung KZ, Ritchie CS. Use of opioids and other analgesics by older adults in the United States, 1999-2010. Pain Med 2015;16:319-27.

4. Gloth FM, 3rd. Pain management in older adults: prevention and treatment. J Am Geriatr Soc 2001;49:188-99.

5. AGS Panel on Persistent Pain in Older Persons. The management of persistent pain in older persons. J Am Geriatr Soc 2002;50:S205-S224.

6. Van den Beuken-van Everdingen M, De Rijke J, Kessels A, Schouten H, Van Kleef M, Patijn J. Prevalence of pain in patients with cancer: a systematic review of the past 40 years. Ann Oncol 2007;18:1437-49.

7. Thomas E, Peat G, Harris L, Wilkie R, Croft PR. The prevalence of pain and pain interference in a general population of older adults: cross-sectional findings from the North Staffordshire Osteoarthritis Project (NorStOP). Pain 2004;110:361-8.

8. Reyes-Gibby CC, Aday L, Cleeland C. Impact of pain on self-rated health in the community-dwelling older adults. Pain 2002;95:75-82.

9. Platts-Mills TF, Flannigan SA, Bortsov AV, et al. Persistent pain among older adults discharged home from the emergency department after motor vehicle crash: a prospective cohort study. Ann Emerg Med 2016;67:166-76.e1.

10. Classen S. Special issue on older driver safety and community mobility. Am J Occup Therapy 2010; 64:211-4.

11. Veldhuijzen DS, van Wijck AJ, Wille F, et al. Effect of chronic nonmalignant pain on highway driving performance. Pain 2006;122:28-35.

12. Fan A, Wilson KG, Acharya M, Cranney A, Buenger U, Marshall S. Self-reported issues with driving in patients with chronic pain. $P M \& R$ 2012;4:87-95.

13. Forrest KYZ, Bunker CH, Songer TJ, Coben JH, Cauley JA. Driving patterns and medical conditions in older women. J Am Geriatr Soc 1997;45:1214-8.

14. Sargent-Cox KA, Windsor T, Walker J, Anstey KJ. Health literacy of older drivers and the importance of health experience for self-regulation of driving behaviour. Accid Anal Prev 2011;43:898-905.

15. Chihuri S, Mielenz T, DiMaggio C, et al. Driving cessation and health outcomes in older adults. Washington, DC: AAA Foundation for Traffic Safety; 2015

16. Harrison A, Ragland DR. Consequences of driving reduction or cessation for older adults. Transportation Research Record 2003;1843:96-104.

17. Gostin LO, Hodge JG, Jr, Noe SA. Reframing the opioid epidemic as a national emergency. JAMA 2017;318:1539-40.
18. Lagarde E, Chastang JF, Lafont S, Coeuret-Pellicer $M$, Chiron M. Pain and pain treatment were associated with traffic accident involvement in a cohort of middle-aged workers. J Clin Epidemiol 2005;58:524-31.

19. Chihuri S, Li G. Use of prescription opioids and initiation of fatal 2-vehicle crashes. JAMA Netw Open 2019;2:e188081.

20. Chihuri S, Li G. Use of prescription opioids and motor vehicle crashes: a meta analysis. Accid Anal Prev 2017;109:123-31.

21. Lenné MG, Dietze P, Rumbold G, Redman JR, Triggs TJ. Opioid dependence and driving ability: a review in the context of proposed legislative change in Victoria. Drug and Alcohol Review 2000;19:427-39.

22. Rudisill TM, Zhu M, Kelley GA, Pilkerton C, Rudisill BR. Medication use and the risk of motor vehicle collisions among licensed drivers: a systematic review. Accid Anal Prev 2016;96:255-70.

23. Hetland A, Carr DB. Medications and impaired driving: a review of the literature. Ann Pharmacother 2014;48:494-506.

24. American Geriatrics Society. Beers Criteria Update Expert Panel. American Geriatrics Society 2015 updated Beers Criteria for potentially inappropriate medication use in older adults. J Am Geriatr Soc 2015;63:2227-46.

25. Li G, Eby DW, Santos R, et al. Longitudinal Research on Aging Drivers (LongROAD): Study design and methods. Inj Epidemiol 2017;4:22 .

26. Callahan CM, Unverzagt FW, Hui SL, Perkins AJ, Hendrie HC. Six-item screener to identify cognitive impairment among potential subjects for clinical research. Med Care 2002;40:771-81.

27. Craig BM, Reeve BB, Brown PM, et al. US valuation of health outcomes measured using the PROMIS-29. Value Health 2014;17:846-53.

28. Dowell D, Haegerich TM, Chou R. CDC guideline for prescribing opioids for chronic pain-United States. MMWR Recomm Rep 2016;65:1-49.

29. Department of Health and Human Services. 2018 Annual surveillance report of drug-related risks and outcomes-United States. 2018. Available from: https://www.cdc.gov/drugoverdose/pdf/pubs/2018cdc-drug-surveillance-report.pdf. Accessed June 5, 2019.

30. Chou R, Deyo R, Devine B, et al. The effectiveness and risks of long-term opioid treatment of chronic pain. Evid Rep Technol Assess (Full Rep) 2014;1-219.

31. Papaleontiou M, Henderson CR, Jr, Turner BJ, et al. Outcomes associated with opioid use in the treatment of chronic noncancer pain in older adults: a systematic review and meta-analysis. J Am Geriatr Soc 2010;58:1353-69. 